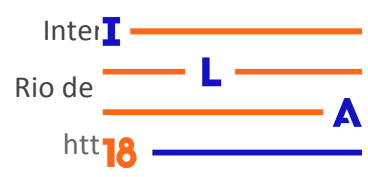

\title{
UN ACERCAMIENTO TEÓRICO SOBRE LA PROBLEMÁTICA DEL DISEÑO DE APLICACIONES MÓVILES PARA UNA POBLACIÓN URBANA.
}

\section{A THEORETICAL APPROACH ON THE PROBLEM OF THE DESIGN OF MOBILE APPLICATIONS FOR AN URBAN POPULATION.}

\author{
Griselda Alejandra Molina ${ }^{1}$, Esp. \\ (1) Universidad de Buenos Aires \\ e-mail:alejandramolina88@gmail.com
}

\begin{abstract}
Diseño de interacción en gobiernos, Modelo teórico, Modelo de usuario El trabajo se centra en el análisis del diseño de interacción en aplicaciones móviles, dirigidas a la población urbana por parte del gobierno.
\end{abstract}

Interaction design in government, Theoretical model, User model

The work focuses on the analysis of interaction design in mobile applications, aimed at the urban population by the government.

\section{Introducción}

En está última década, creció de manera exponencial la forma en que se implementa el diseño de experiencia de usuario en proyectos de índole estatal. Como las nuevas tecnologías ofrecen un mejor acercamiento a los ciudadanos, los gobiernos la utilizan cada vez más para brindar servicios que mejoren la calidad de vida de los habitantes.

Por esta razón, en este escrito se pretenderá echar luz sobre un área de vacancia: la metodología de diseño de experiencia de usuario implementado por gobiernos en aplicaciones móviles en poblaciones urbanas.

Los casos de estudio elegidos son dos aplicaciones móviles del Gobierno de la Ciudad de Buenos Aires entre los años 2015 y 2016. La primera app mencionada permite al usuario moverse con diferentes medios de transportes por la ciudad (BA Cómo Llego); y la otra está dirigida al control de las embarazadas (BA Embarazo).

Se establecerán los principales parámetros para analizar de una manera crítica en el marco de la teoría de diseño de experiencia, la interacción de los productos digitales y su relación con la heterogeneidad que presenta la población de la ciudad mencionada.

En las próximas páginas se hará una breve reseña de las investigaciones actuales que tocan algunas aristas de la problemática a analizar. Luego se introducirá la teoría de diseño de experiencia de usuario en profundidad para confrontar sus conceptos con otros autores de teoría del diseño $\mathrm{y}$ de medios.

Asimismo, se presentarán y analizarán los casos de estudio desde un enfoque teórico; y por último se hará una conclusión con los factores que podrían ser relevantes a la problemática.

\section{Estado de la cuestión}

\subsection{Mapeo general de la metodología del diseño centrado en el usuario en gobiernos}

Entre las décadas de 1990 y 2010 el diseño de experiencia de usuario tuvo un desplazamiento del diseño de objetos al de interfaces. En los últimos tiempos esta metodología pasó del diseño de objetos industriales al diseño de interfaces digitales para el Estado, entre otros ámbitos.

Es ahí donde las aplicaciones móviles para celulares juegan un rol importante, porque los gobiernos intentan mejorar la manera en que ofrecen servicios o tratan de vincularse con los habitantes. En la actualidad, las investigaciones que existen sobre el diseño de interacción y el gobierno tienen planteado varios ejes temáticos. 


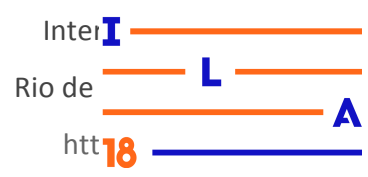

\section{Diseño de interacción accesible}

En el diseño de interacción accesible, los padres de la metodología del diseño de experiencia de usuario Nielsen y Norman, hacen mención a las reglas del diseño "accesible" como solución a la inclusión digital de personas con capacidades diferentes.

Explican que la usabilidad puede mejorar sustancialmente la problemática haciendo que los sitios web y las intranets apoyen el desempeño de las tareas para los usuarios con discapacidades (NIELSEN, 2001).

También afirman que no solo el diseño accesible permite la mejor navegabilidad de personas con discapacidad, sino que mejora exponencialmente la navegación las personas en general. En un estudio dónde midieron la efectividad de la navegación de páginas accesibles, se detectó que "de hecho, los usuarios con mayor nivel de alfabetización también obtuvieron calificaciones más altas en las tres métricas de usabilidad al usar el sitio revisado" (NIELSEN, 2005).

Es además optimista con la problemática de la brecha económica, no así con la brecha digital. Esto es porque ve menores progresos en el diseño de usabilidad para las personas con menos habilidades tecnológicas. Reconoce que se intuye cómo mejorar la interactividad, pero por alguna u otra razón no se está haciendo.

\section{Comunicación digital interactiva}

Scolari cuestiona la simplificación de soluciones que el diseño de experiencia de usuario propone paramejorar la interacción. Afirma que la red [o el producto digital] no es instrumento de producción, sino un ambiente de comunicación e interacción que genera muchos conflictos que no se solucionan de una sola manera (SCOLARI, 2004).

Asimismo, recomienda aplicar conceptos teóricos de semiótica para solucionar otros aspectos que el diseño de interacción no abarca, pero que son necesarios.

La incorporación de un concepto cooperativo y conflictivo de los procesos de interacción hombre-computadora ayudaría a los investigadores a ir más allá de las concepciones instrumentales ("interfaz como una prótesis que desaparece" e "interacción hombre-computadora como un proceso transparente"). La semiótica, en este contexto, podría ser muy útil para deconstruir esta visión superficial de las interacciones y mejorar el análisis de las interfaces digitales para ir más allá de la tradición de la investigación de usabilidad"

\section{Inclusión digital en gobiernos}

Con relación a la inclusión digital en gobiernos, el cuestionamiento gira en torno a los beneficios de la tecnología cívica y si realmente mejora la cotidianidad de los habitantes.

Rumbul cree que es positivo, porque sostiene que las nuevas tecnologías cívicas tienen que venir acompañadas de investigaciones sobre el contexto específico donde van a ser implementadas. También considera que hay una diferencia en la manera que reciben los nuevos servicios tecnológicos entre los países del centro y de la periferia:

Los datos demográficos básicos de los usuarios de la tecnología cívica en los países en desarrollo apuntan a un grupo más joven y menos educado que domina las plataformas de participación en línea [...] Existe un riesgo significativo de exclusión digital de los ciudadanos pertenecientes a las generaciones mayores y de los individuos residentes en zonas de limitada conectividad [...] Esta exclusión puede hacer que ciertos grupos de la sociedad no tengan voz y, por lo tanto, las políticas no puedan satisfacer sus necesidades específicas. (RUMBUL, 2015, p.20)

También Asgarkhani (2008) afirma que los esfuerzos deben conducirse no sólo en mejorar la infraestructura tecnológica sino "también en la estructura social, los valores y actitudes culturales, las consideraciones éticas y la cultura política junto con los gobiernos".

Por otro lado, está la problemática de descifrar la relación que hay entre las desventajas tecnológicas y sociales.

Helsper (2008) explica que estas desventajas están "inquebrantablemente conectadas y, que las políticas sociales digitales, serán menos posibles de realizar si no hay una mejora en la manera de educar 


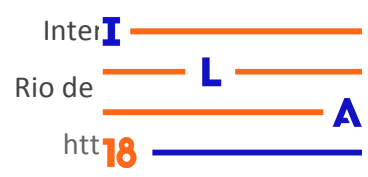

digitalmente". Si esto no se tiene en cuenta las personas con desventajas económicas también estarán marginadas digital y socialmente.

Mulgan tampoco es optimista con la implementación de la innovación a través de metodologías de diseño en sectores públicos, en especial en gobiernos:

por un lado, los gobiernos están bien situados para aumentar las ideas; por otro lado, el sector público es notoriamente pobre en la adopción de nuevas ideas, incluso cuando están sustentados por evidencia sólida”. (MULGAN 2014, p.13)

Por último, se encontró una investigación sobre innovación de ciudades en Latinoamérica. Bolay, Pedrazzini, Rabinovich, Catenazzi and Pleyan cuestionan la efectividad de la aplicación de innovación social en esta región.

Para ellos, el carácter innovador depende del potencial para mejorar significativamente la calidad de vida de todas las partes involucradas, sobre todo de los más pobres. "La innovación, sea cual sea su valor añadido técnico, no es más que un epifenómeno si no refuerza la sostenibilidad del desarrollo tanto ambiental como social" (BOLAY ET AL, 2005).

Sin embargo, lo particular de el caso de estudio sobre la La Paz y Buenos Aires, fue que en estos lugares encontraron una "gran inventiva" para tratar de solucionar problemas sociales.

Es importante apreciar esta creatividad, atrapada entre las limitaciones externas y la voluntad de "encontrar una solución" en un mundo globalizado que tiende a aumentar las desigualdades no sólo entre los países y las regiones del mundo, sino también dentro de las sociedades industrializadas. (LATOUCHE, 2000) (BOLAY ET AL. 2005, p.15)

\subsection{La metodología del diseño centrado en el usuario en Buenos Aires}

En conclusión, hay información, pero dispersa sobre las diferentes aristas que tocan a la problemática del diseño centrado en el usuario cuando se utiliza en gobiernos.

Faltan investigaciones específicas en las áreas de diseño de aplicaciones en gobiernos latinoamericanos y más todavía, cuando se trata de diseñar para una población específica.

Por lo tanto, es posible y acertado proponer un análisis sobre un gobierno de una ciudad latinoamericana como Buenos Aires, y la manera en la que abordan el diseño de aplicaciones móviles a través del diseño de interfaces.

\section{Marco teórico}

\subsection{Definición de ejes conceptuales}

Como el trabajo está enfocado en realizar un primer acercamiento al análisis teórico de la problemática de la interacción, es necesario plantear algunos parámetros que sirvan de ejes conceptuales. Estos ejes serán sobre teoría de diseño y teoría de los medios.

\subsection{Teoría del diseño centrado en el usuario según Norman}

Primero es importante definir las áreas de la teoría del diseño que escriben sobre el tema. Para entender la metodología analizada, se citará a Donald Norman por ser uno de los referentes teórico que más se referencia en el ambiente laboral, y no solo en el académico.

En su libro La psicología de los objetos cotidianos, él analiza los aspectos del diseño de experiencia de usuario, proponiendo las primeras definiciones teóricas de esta disciplina.

\section{Definición del diseño de experiencia de usuario}

Norman (1988) define el diseño de experiencia de usuario como:

Una teoría basada en las necesidades y los intereses del usuario, con especial hincapié en hacer que los productos sean utilizables y comprensibles. [...] El diseño debería:

- Facilitar la determinación de qué actos son posibles en cada momento dado (utilizar limitaciones).

- Hacer que las cosas sean visibles, comprendido el modelo conceptual del sistema, los diversos actos posibles y los resultados de esos actos.

- Hacer que resulte fácil evaluar el estado actual del sistema. 


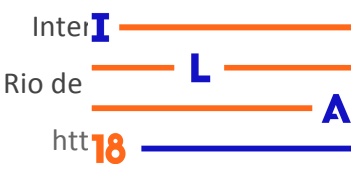

- Seguir las topografías naturales entre las intenciones y los actos necesarios; entre los actos $\mathrm{y}$ el efecto consiguiente, y entre la información que es visible y la interpretación del estado del sistema.

El diseño de experiencia de usuario, según este autor, trata de acercarse a la solución de una problemática analizando las necesidades, actos y conocimientos del usuario. Teniendo estos factores en cuenta, el diseño debería minimizar al máximo los errores o las malas interacciones con el producto diseñado.

En las próximas páginas se desarrollan los conceptos que forman parte de esta teoría.

\section{Modelos conceptuales y mentales}

Norman introduce los conceptos de modelos conceptuales y mentales que garantizan una buena interacción entre el usuario y el objeto.

Estos conceptos están divididos en modelo de diseño, de usuario e imagen del sistema, donde el

diseñador espera que el modelo del usuario sea idéntico al modelo de diseño. Pero el diseñador no habla directamente con el usuario: todas las comunicaciones se realizan por conducto de la imagen del sistema. Si la imagen del sistema no hace que el modelo de diseño sea claro y coherente, entonces el usuario acabará con el modelo mental equivocado. (NORMAN, 1986 Citado en NORMAN 1998, p.32)

Esta manera que tiene el autor de definir los modelos conceptuales y mentales también se ha puesto en cuestionamiento últimamente. Esto se planteará con más detenimiento cuando se presente la opinión de Scolari.

\section{Principios de interacción}

La interacción entre el usuario y el objeto deber ser lo menos problemática posible, para poder garantizar un buen diseño. El autor enumera varios criterios para conocer cómo funcionan las cosas y generar una buena interacción: prestaciones, indicadores, topografías y limitaciones.

El teórico divide estas limitaciones en físicas (las propiedades físicas del objeto), semánticas (remiten al conocimiento de la situación y el mundo), lógicas (la relación entre las disposición espacial o funcional de los componentes y las cosas a las que éstos afectan o por las que se ven afectados) y culturales/sociales.

De todas las limitaciones, las culturales y sociales no aparecen bien explicadas en la teoría. Explica que las limitaciones sociales son conjuntos de actos que son considerados permisibles para las situaciones sociales de una cultura determinada. En su libro, no se especifica cómo influye las divisiones socioculturales de una misma población en la construcción de la interacción.

El tema de la influencia que tiene lo social y el contexto al implementar una metodología, permite pasar al punto siguiente del análisis, que son las contraposiciones de otros teóricos ante los conceptos específicos que utiliza el autor.

\subsection{Confrontaciones de otros autores ante la teoría de Norman}

\section{Alexander y el ajuste}

Según Norman (1998), el diseño para poder tener interacciones exitosas debe utilizar las propiedades naturales de la gente y del mundo: debe explotar las relaciones naturales y las limitaciones naturales que lo vinculan con los objetos.

Contrariamente, Alexander (1976) introduce el concepto de ajuste o fitness para explicar que la construcción del diseño de interacción no es natural, sino que es una negociación entre la forma y el contexto.

Cuando hablamos de diseño, el objeto real de la discusión no es sólo la forma sino el conjunto que comprende la forma y su contexto. El eficaz ajuste es una propiedad deseable de este conjunto

que la relaciona con alguna división particular del conjunto en forma y contexto (ALEXANDER, 1976, p.22-23).

La tarea del diseñador entonces es poder diseñar sobre la forma, que es la parte del conjunto sobre la cual se puede ejercer control, conociendo cual es el contexto al que se están enfrentando. 


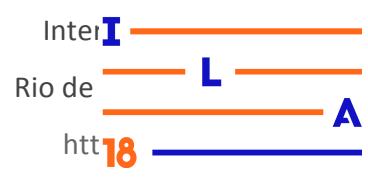

Por eso es sumamente importante tener en cuenta donde está situado el usuario contextualmente, para poder ver sus necesidades y la manera en la que se relaciona con el objeto de diseño a través de la cultural en la que habita para proyectar interacciones "exitosas".

\section{Manzini y el localismo cosmopolita}

Siguiendo con el concepto de contexto Manzini, en oposición a Norman, es consciente de las diferencias sociales y culturales que hay en utilizar una metodología pensada para un contexto específico. Él propone adecuar las metodologías a las características concretas del entorno cultural y funcional:

Creo en la importancia de esta actividad de interpretación y adaptación: la transmisión hacia la sostenibilidad puede y debe verse como un gran proceso de aprendizaje social en el que es también necesario aprender de cuanto sucede en otras partes del mundo" (MANZINI, 2015, p.8).

Asimismo, explica que al tener que diseñar proyectos que afecten a una población de una sociedad determinada se debe tener en cuenta la noción de lo "local", haciendo un balance entre conservar lo propio del lugar sin ser hermético y mostrarse abierto a los flujos globales de planteamientos y metodologías.

Utiliza el concepto de localismo cosmopolita para referirse a esta nueva manera de ver lo local como una "cultura de una sociedad en la que los lugares y las comunidades no son entidades aisladas, sino que se convierten en nodos de una gran diversidad de redes" (MANZINI, 2015).

\section{Julier y el alto diseño}

Siguiendo con la línea conceptual de Manzini sobre la importancia del contexto, se cuestiona si las metodologías creadas en un país del centro (como Estados Unidos), también puede implementarse exitosamente en países de la periferia.

Sería interesante traer el concepto de alto diseño de Julier para explicar este conflicto. Para el autor, el alto diseño oculta los procesos de producción, para "hacer que parezca que la estabilidad y la inestabilidad surgen del propio producto, y no de los agentes que los moldean y presentan en formas particulares" (JULIER, 2010).

Esto podría hacer que implementar una metodología de otro contexto sea contraproducente, porque si algo no sale bien, generalmente se examina el resultado y no cómo (o quiénes) lo construyeron.

Julier además explica que el contexto y el sistema económico en el que se creó ciertamente influyen.

"Cualquier acto de oscuridad deliberada o cualquier negación consciente dentro del alto diseño nos dice algo del contexto más amplio de la creación de valor" (JULIER, 2010, p.121).

\section{Scolari, el contrato de interacción y el modelo de usuario}

Continuando con la problemática de aplicar una metodología en contextos específicos, Scolari (2004) declara que "el investigador debe desembarazarse de los marcos institucionales que decretan $<<$ normas de empleo $>>$ y considerar con mayor atención las situaciones reales de uso".

Por ese mismo motivo, se opone a la idea de modelo de usuario de Norman, ya que la imagen del sistema no hace que el usuario se equivoque. Es el diseñador quien tiene la responsabilidad de corresponder lo que piensa qué hará el usuario y lo que terminará haciendo.

También se debe tener en cuenta el contrato de interacción, que es un término de Scolari similar al contrato de lectura de Verón. Este concepto explica que el autor o diseñador debe construir el texto para un lector o usuario específico, quien puede rechazarlo si no se ve reflejado o representado. “Aceptar un contrato de interacción significa para el usuario entrar en un mundo con su propia gramática" (SCOLARI, 2004, p.157).

Por lo tanto, el problema no radicaría en lo que el usuario entienda o en lo que el dispositivo digital muestre, sino en la manera en que el diseñador aplique (de manera crítica) la teoría en la construcción del diseño. 


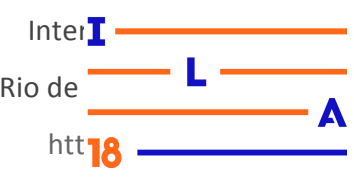

\section{Metodología}

Corpus

-Proyecto de la aplicación BA Mi Embarazo

(enfoque principal del trabajo).

-Aplicación BA Cómo Llego (enfoque secundario comparativo).

Lugar

-Buenos Aires, años 2015 y 2016

El estudio está realizado desde un enfoque cualitativo y se busca realizar un primer acercamiento exploratorio teórico a la problemática de la experiencia de usuario, a través del discurso y de la funcionalidad de las apps.

El corpus está formado por dos aplicaciones móviles del Gobierno de la Ciudad de Buenos Aires. La elección de estas se debe a que están producidas por el mismo gobierno y dirigidas a la población de esa ciudad.

\section{Análisis de las aplicaciones móviles BA Embarazo y BA Cómo Llego}

\section{Introducción a la problemática}

Este análisis se centró en la Ciudad de Buenos Aires, Argentina; entre los años 2015- 2016 sobre las aplicaciones móviles que realiza su gobierno. Esta ciudad tiene casi 3 millones de habitantes y concentra la mayor parte de las actividades económicas, políticas, sociales, turísticas y culturales del país.

Es en este contexto en el que transcurre el auge de las aplicaciones móviles para celulares inteligentes. De esta manera, BA Cómo Llego y BA Embarazo surgen como políticas destinadas a la modernización tecnológica de cara a los ciudadanos, de movilidad en el primer caso y de salud en el segundo.

App BA Cómo Llego, el primer caso de estudio, es una aplicación para celulares inteligentes y computadoras que permite al usuario trasladarse a través de indicaciones y con un mapa por toda la ciudad. Es usada tanto por gente que está de paso como por los mismos ciudadanos, esto se debe a las grandes distancias que tiene esta ciudad, una superficie total de 203 kilómetros.
En cambio, la app BA Embarazo, el segundo caso de estudio, es un encargo del Ministerio de Salud específico para mejorar el control las de embarazadas, en especial de las mujeres porteñas de bajos recursos que no tienen acceso a la información necesaria en la etapa de gestación.

Esta aplicación permite sacar turnos con obstetras de todos los centros de salud públicos de la ciudad, anotar otro tipo de citas y conocer qué ocurre en cada una de las semanas en el cuerpo de la mujer y el bebé.

Según datos del Análisis de Situación de Salud de esa ciudad, "para el año 2016, un 27\% de los embarazos registrados no accede a los controles suficientes durante el embarazo y un 5\% no han tenido contacto con el sistema hasta el momento del parto".

Más adelante, se hará una comparación teórica entre estas aplicaciones.

\section{Los modelos teóricos y su aplicación en la realidad}

Volviendo a los modelos teóricos, Norman (1998), explica que la brecha que hay entre la teoría y la realidad es predecible, porque las limitaciones físicas son pocas. Pero si observamos los casos de estudio, también se podrían encontrar limitaciones según el nivel socio-económico de los usuarios de una misma población.

Los habitantes de clase media y alta podrían tener menos inconvenientes en usar ambas aplicaciones por contar con los recursos necesarios cubiertos (monetarios y de infraestructura). En cambio 

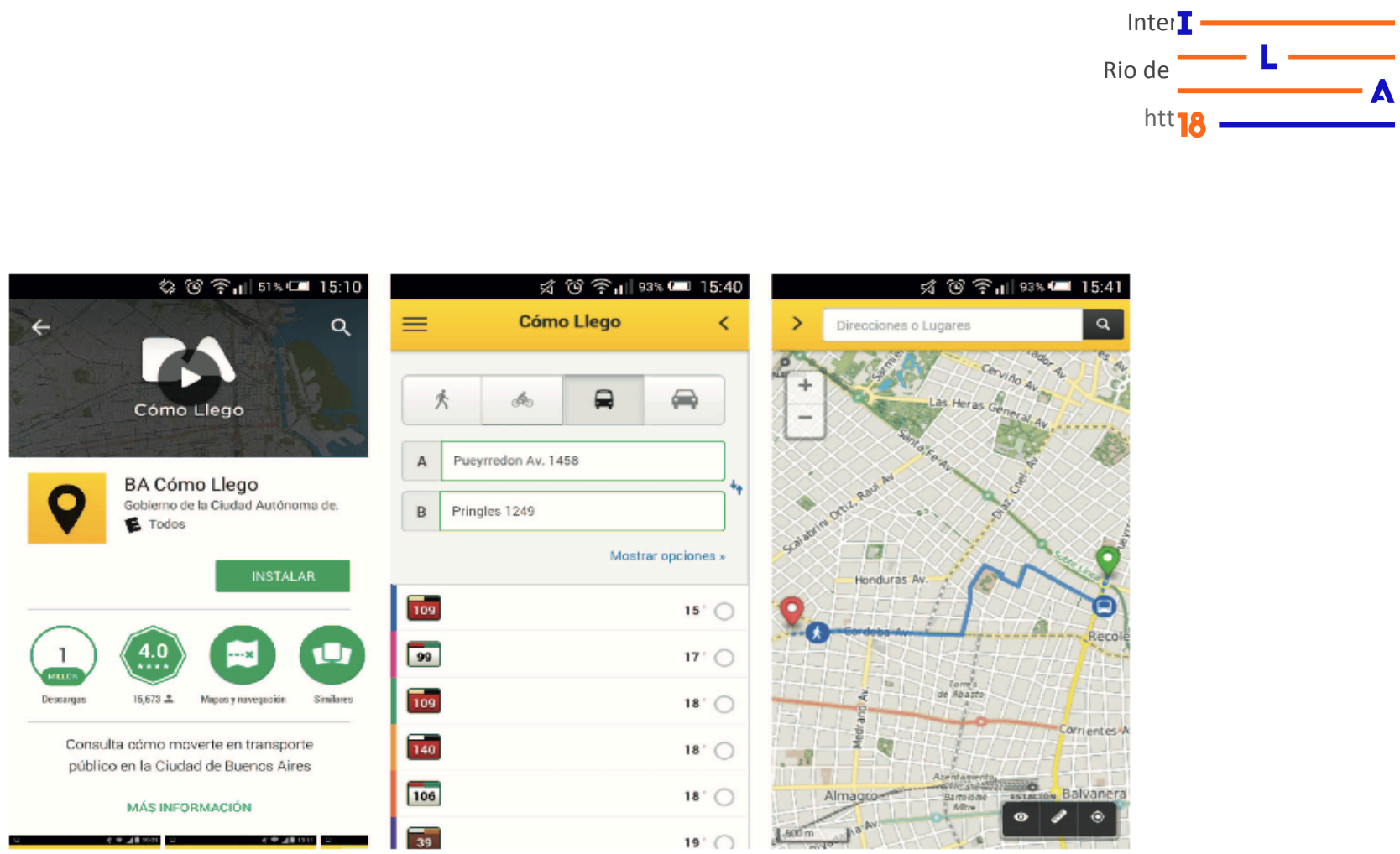

Figura 1. Aplicación móvil BA Cómo Llego (Version 4.2.0). Buenos Aires. Primera imagen (de izquierda a derecha): presentación de la aplicación en Google play, lugar donde se descargan las apps para usarse en el sistema operativo Android. Segunda imagen: Detalle de la aplicación en uso. Tercera imagen: Detalle del mapa de la aplicación en uso. Fuente: Capturas de pantalla, elaboración propia (2017).
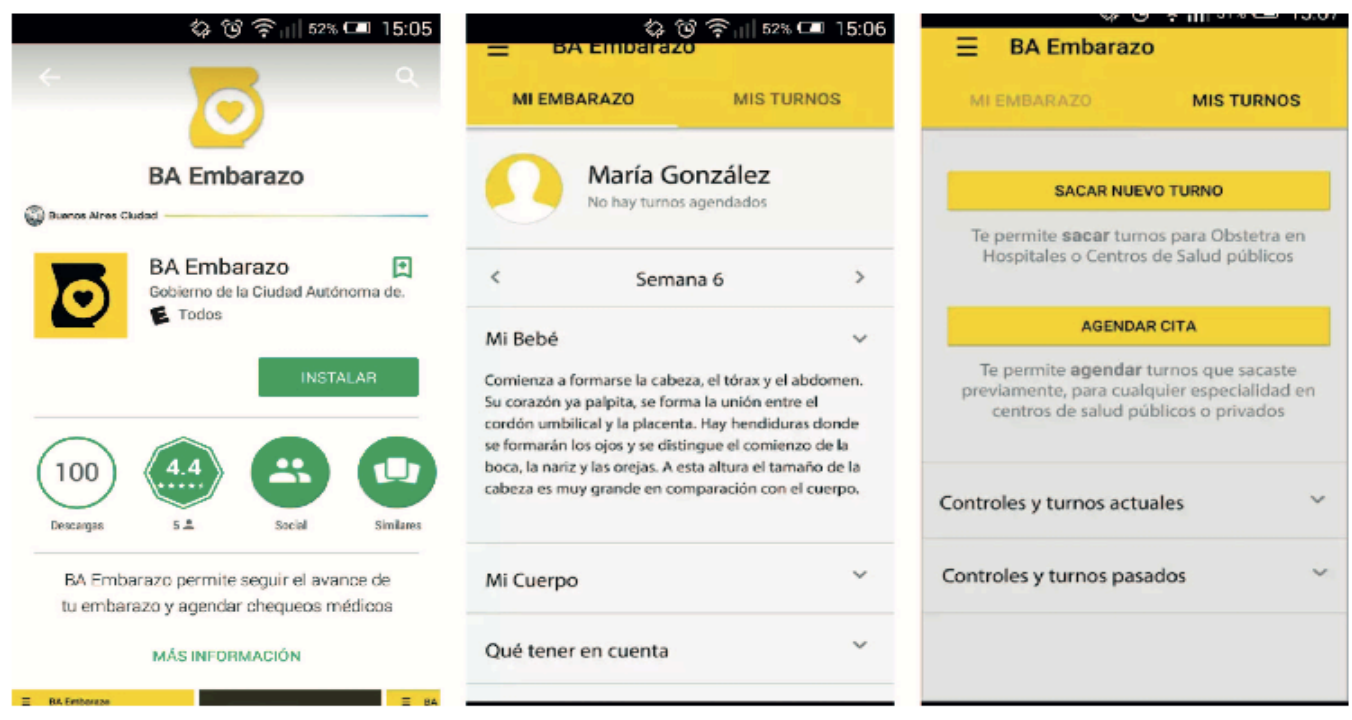

Figura 2. Aplicación móvil BA Embarazo (Version 1.0). Buenos Aires. Primera imagen (de izquierda a derecha): presentación de la aplicación en Google Play, lugar donde se descargan las apps para usarse en el sistema operativo Android. Segunda imagen: Detalle de las funcionalidades de la aplicación en uso, seguimiento de las semanas de embarazo. Tercera imagen: Detalle del mapa de la aplicación en uso, botones para acceder a turnos en centros de 


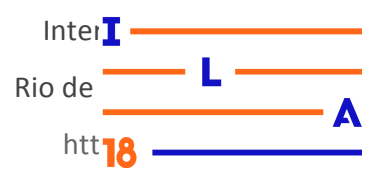

aquellos de bajos recursos estarían más limitados en el uso de estas apps, por contar con menos recursos, como la calidad de sus celulares y el escaso acceso a internet en asentamientos informales.

Scolari (2004), a comparación de Norman, advierte que los modelos teóricos por más o menos semióticos que sean, "presentan siempre una serie de limitaciones que es conveniente enumerar antes de comenzar a trabajar con ellos".

Una de estas limitaciones es que los modelos al ser una hipótesis, exigen una permanente verificación empírica, porque estos no están garantizados de porvida. También explica que la teoría no puede ser confundida con la realidad ni muchos menos pretender sustituirla, "como máximo pueden brindar una explicación -siempre hipotética-del funcionamiento de algún aspecto de la misma" (Scolari, 2004, p.148).

\section{La construcción del modelo de usuario}

Algo similar a la problemática de la teoría podría ocurrir con la forma en que se construye el modelo de usuario. Según Norman (1988), el modelo de usuario es la [idea] "que elabora el usuario para explicar el funcionamiento del sistema. Idealmente, el modelo del usuario y el del diseño son equivalentes".

El diseñador debe asegurar que el sistema revele la imagen idónea del sistema. Es la única forma de que el usuario pueda adquirir el modelo correcto del usuario y encontrar apoyo para que las intenciones queden reflejadas en actos y el estado del sistema en interpretaciones. Debe recordarse que el usuario adquiere todos sus conocimientos del sistema a partir de esa imagen del sistema (NORMAN, 1988, p. 235)

El problema de esta premisa sería cómo hace el diseñador para armar un único modelo que abarque la heterogeneidad de usuarios en una población urbana (con sus diferencias socioeconómicas incluídas).

Scolari, contrariamente a lo que expresa Norman, está en desacuerdo en la construcción un único modelo de usuario para todas las personas. Él trae la noción de lector modelo de Eco y lo asemeja al concepto usuario modelo, para explicar que diseñar para el lector modelo "no significa sólo esperar que exista, significa también mover el texto para construirlo" (ECO, 1979 en SCOLARI, 2004, p.157).

Teniendo en cuenta la noción del modelo de usuario, en los casos de estudio, se puede examinar que la manera en la que aparece el contenido las aplicaciones difiere bastante. Se observa un predominio mayor de imagen en BA Cómo Llego, siendo el mapa y los iconos fácilmente reconocibles, por ser los elementos más importantes con los que el usuario interactúa en este tipo de app.

En BA Embarazo en cambio, se advierte un exceso de indicaciones textuales. Se puede apreciar que hay texto de acompañamiento debajo de cada uno de los de botones "Sacar turno" y "Agendar cita".

Generalmente el texto de acompañamiento o explicativo en una aplicación móvil suele ser más corto, o incluso solo presentarse la primera vez que el usuario abre la app (como en el onboarding). Pero en este caso particular presumiblemente, se eligió que esté presente en todo momento para que el usuario no sólo recuerde, sino que tenga visible las acciones que puede realizar.

Pareciera que se tuvo en cuenta en el diseño el entorno social de las mujeres a las que está dirigida la aplicación. No se analiza si fue la mejor manera de elegir los componentes de interacción que requiere el proyecto, sino resaltar que hay indicios visuales que harían suponer que la forma de construir el diseño difiere de la manera "estándar" en la que se diseña para "toda" la población.

En este análisis comparativo, se tiene en cuenta que las aplicaciones tienen funcionalidades diferentes, y que por eso la forma de mostrar el contenido es distinta. Aún así, lo que se intenta es dilucidar si la desigualdad en la proporción de texto e imagen connotarían más de un tipo de modelo de usuario en la misma población que forma parte de esa ciudad.

\section{Conclusiones}

En resumen, al ser una investigación exploratoria, en este trabajo se propuso empezar a abordar la problemática de la inecuación entre diseño y uso en aplicaciones móviles para una población urbana por parte del gobierno. 


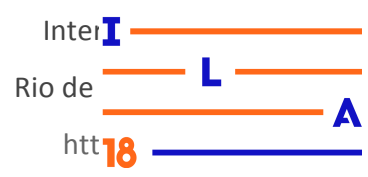

A partir de la presentación de los casos y el marco teórico, se conocieron los factores que podrían ser relevantes al problema. Estos serían la aplicación a la realidad de los modelos teóricos y la construcción de modelos de usuarios.

Por lo tanto, sería interesante profundizar en estos factores y analizarlos en otras investigaciones más complejas como ser métricas, entrevistas contextuales u otro tipo de método cuantificable o medible.

Además, convendría estudiar cómo influye el contexto socio-económico en la usabilidad de estos tipos de aplicaciones, en Buenos Aires u otra ciudad latinoamericana.

\section{Referencias bibliográficas}

Análisis de Situación de Salud de la Ciudad Autónoma de Buenos Aires - Año 2016. Web de Información Epidemiológica del Gobierno de la Ciudad Autónoma de Buenos Aires, 2016. Disponible en:

$<$ http://www.buenosaires.gob.ar/salud/analisisdesituacion-de-salud $>$. Acceso en el año 2017.

ALEXANDER, Christopher. Ensayo sobre la Síntesis de la forma. Ediciones Infinito. Buenos Aires, 1976

\section{ASGARLHANI, Mehdi. The Reality of Social} Inclusion Through Digital Government, Journal of Technology in Human Services. 25 (1-2), 127146, 2004. Disponible en:

$<$ http://icter.org/conference/sites/default/files/icter/I CEG-2004p7.pdf >. Aceeso en el año 2017

BOLAY, J.C.; PEDRAZZINI, Y.; RABINOVICH, A.; CATENAZZI, A.; PLEYAN, C. G. Urban environment, spatial fragmentation and social segration in Latin America: where does innovation lie? Habitat International. 29, 627-645. (2005). Disponible en:

$<$ https://infoscience.epfl.ch/record/128235?of=HB >. Acceso en el año 2017.

HELSPER, Ellen. Digital inclusion: an analysis of social disadvantage and the information society. Department for Communities and Local Government. Londres, 2008. Disponible en: $<$ http://eprints.lse.ac.uk/26938/1/_libfile_REPOSIT ORY_Content_Helsper,\%20E_
Digital\%20inclusion_Helsper_Digital\%20inclusion_ 2013.pdf $>$. Acceso en el año 2017

JULIER, Guy. La cultura del diseño. Gustavo Gili. Barcelona, 2010.

MANZINI, Ezio. Cuando todos diseñan. Una introducción al diseño para la innovación social. Experimenta. Madrid, 2015.

\section{MULGAN, Geoff. Innovation in the Public} Sector, how can public organizations better create, improve and adapt? Londres, 2014. Disponible en:

$<$ http://www.nesta.org.uk/publications/innovationpu blic-sector $>$. Acceso en el año 2017

NIELSEN, Jakob. (2005). Beyond Accessibility: Treating Users with Disabilities as People. California, EU.: Nielsen Norman Group. Disponible en:

$<$ https://www.nngroup.com/articles/beyondaccessibi lity-treating-users-with- disabilities-aspeople/>.

Acceso en el año 2017.

NIELSEN, Jakob. Digital Divide: The 3 Stages. Nielsen Norman Group. California, 2006. Nielsen Norman Group. Disponible en:

$<$ https://www.nngroup.com/articles/digitaldividethe-three- stages/ >. Acceso en el año 2017.

NIELSEN, Jakob. Lower-Literacy Users: Writing for a Broad Consumer Audience. Nielsen Norman Group, California, 2005. Disponible en:

$<$ https://www.nngroup.com/articles/writingforlower-literacy-users/>. Acceso en el año 2017.

\section{NORMAN, Donald. La psicología de los objetos} cotidianos. NEREA. Madrid, 1998.

RUMBUL, Rebecca. Who benefits from civic technology? Demographic and public attitudes research into the users of civic technologies. 2015. Disponible en:

$<$ https://www.mysociety.org/research/whobenefitsfrom-civic-technology/>. Acceso en el año 2017.

\section{SCOLARI, Carlos. Digital Eco_Logy: Umberto} Eco and a semiotic approach to digital communication. Information, Communication \& Society. (12:1), pp.129- 148. 2009. Disponible en: $<$ http://www.tandfonline.com/doi/abs/10.1080/1369 $1180802520798>$. Acceso en el año 2017. 


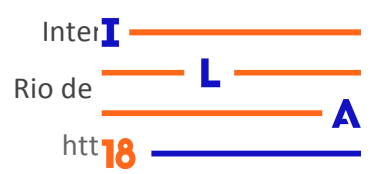

SCOLARI, Carlos. Hacer clic. Hacia una

sociosemiótica de las interacciones digitales.

Editorial Gedisa. Barcelona, 2004.

SCOLARI, Carlos. Los usos de Nielsen. Para una crítica de la ideología de la usabilidad. Scribd, 2001. Disponible en:

$<$ https://es.scribd.com/document/31713415/LOSUS OS-DE-NIELSEN-Para-una-critica-de-laideologiade-la-usabilidad\#>. Acceso en el año 2017.

\section{Agradecimientos}

A mi familia, amigos, compañeros de trabajo y de estudio por su apoyo constante. También a la UBA su educación pública, gratuita y de calidad. 